PROCEEDINGS OF THE AMERICAN MATHEMATICAL SOCIETY

Volume 125, Number 5, May 1997, Pages 1487-1495

S 0002-9939(97)03726-X

\title{
WEAKLY COUPLED BOUND STATES IN QUANTUM WAVEGUIDES
}

\author{
W. BULlA, F. GESZTESY, W. RENGER, AND B. SIMON
}

(Communicated by Palle E. T. Jorgensen)

\begin{abstract}
We study the eigenvalue spectrum of Dirichlet Laplacians which model quantum waveguides associated with tubular regions outside of a bounded domain. Intuitively, our principal new result in two dimensions asserts that any domain $\Omega$ obtained by adding an arbitrarily small "bump" to the tube $\Omega_{0}=\mathbb{R} \times(0,1)$ (i.e., $\Omega \supsetneqq \Omega_{0}, \Omega \subset \mathbb{R}^{2}$ open and connected, $\Omega=\Omega_{0}$ outside a bounded region) produces at least one positive eigenvalue below the essential spectrum $\left[\pi^{2}, \infty\right)$ of the Dirichlet Laplacian $-\Delta_{\Omega}^{D}$. For $\left|\Omega \backslash \Omega_{0}\right|$ sufficiently small (|.| abbreviating Lebesgue measure), we prove uniqueness of the ground state $E_{\Omega}$ of $-\Delta_{\Omega}^{D}$ and derive the "weak coupling" result $E_{\Omega}=\pi^{2}-\pi^{4}\left|\Omega \backslash \Omega_{0}\right|^{2}+O\left(\left|\Omega \backslash \Omega_{0}\right|^{3}\right)$ using a Birman-Schwinger-type analysis. As a corollary of these results we obtain the following surprising fact: Starting from the tube $\Omega_{0}$ with Dirichlet boundary conditions at $\partial \Omega_{0}$, replace the Dirichlet condition by a Neumann boundary condition on an arbitrarily small segment $(a, b) \times\{1\}, a<b$, of $\partial \Omega_{0}$. If $H(a, b)$ denotes the resulting Laplace operator in $L^{2}\left(\Omega_{0}\right)$, then $H(a, b)$ has a discrete eigenvalue in $\left[\pi^{2} / 4, \pi^{2}\right)$ no matter how small $|b-a|>0$ is.
\end{abstract}

\section{§1. InTRODUCTION}

Our goal in this paper is to study the bound state spectra of the Dirichlet Laplacian $-\Delta_{\Omega}^{D}$ for open regions $\Omega \subset \mathbb{R}^{n}$ which are tubes outside of a bounded region (quantum waveguides). (Following the traditional notation in quantum physics, we denote the Laplacian by $-\Delta$ as opposed to $\Delta$ in the following.) In particular, let $\Omega_{0} \subset \mathbb{R}^{2}$ be defined by

$$
\Omega_{0}=\mathbb{R} \times(0,1) .
$$

Consider open connected sets $\Omega$ such that:

(i) For some $R>0, \Omega \cap\left\{x \in \mathbb{R}^{2}|| x \mid>R\right\}=\Omega_{0} \cap\left\{x \in \mathbb{R}^{2}|| x \mid>R\right\}$.

(ii) $\Omega_{0} \subset \Omega, \Omega_{0} \neq \Omega$.

Because of condition (i),

$$
\sigma_{\text {ess }}\left(-\Delta_{\Omega}^{D}\right)=\sigma_{\text {ess }}\left(-\Delta_{\Omega_{0}}^{D}\right)=\left[\pi^{2}, \infty\right) .
$$

Then one of our main goals will be to prove

Received by the editors November 13, 1995.

1991 Mathematics Subject Classification. Primary 81Q10, 35P15; Secondary 47A10, 35J10.

Key words and phrases. Dirichlet Laplacians, waveguides, ground states.

This material is based upon work supported by the National Science Foundation under Grant No. DMS-9401491. The Government has certain rights in this material. 
Theorem 1.1. If $\Omega$ obeys (i), (ii), then $-\Delta_{\Omega}^{D}$ has at least one eigenvalue in $\left(0, \pi^{2}\right)$.

Actually, the eigenvalue lies in $\left[\frac{\pi^{2}}{4 R^{2}}, \pi^{2}\right)$ since $\Omega \subset \mathbb{R} \times(-R, R)$ implies

$$
\inf \operatorname{spec}\left(-\Delta_{\Omega}^{D}\right) \geq \inf \operatorname{spec}\left(-\Delta_{\mathbb{R} \times(-R, R)}^{D}\right)=\frac{\pi^{2}}{4 R^{2}} .
$$

We will focus especially on the particular case

$$
\Omega=\Omega_{\lambda},
$$

where

$$
\Omega_{\lambda}=\left\{(x, y) \in \mathbb{R}^{2} \mid 0<y<1+\lambda f(x)\right\}
$$

and $f$ is a $C^{\infty}(\mathbb{R})$ function of compact support with $f \geq 0$. Since $\inf \operatorname{spec}\left(-\Delta_{\Omega}^{D}\right)$ decreases as $\Omega$ increases and every $\Omega$ obeying (i), (ii) has $\Omega_{0} \subset \Omega_{\lambda} \subset \Omega$ for some $f$, it suffices to prove Theorem 1.1 for $\Omega_{\lambda}$ of the form (2). Indeed, it suffices to prove the result for $\lambda$ sufficiently small.

We will prove a much more detailed result in these $\Omega_{\lambda}$ regions for $\lambda$ small enough. Actually, we can replace $f \geq 0$ by the weaker requirement that $\int_{\mathbb{R}} f(x) d x>0$.

Theorem 1.2. Let $\Omega_{\lambda}$ be given by $(2)$ where $f$ is a $C_{0}^{\infty}(\mathbb{R})$ function with $\int_{\mathbb{R}} f(x) d x$ $>0$. Then for all small positive $\lambda,-\Delta_{\Omega_{\lambda}}^{D}$ has a unique eigenvalue $E(\lambda)$ in $\left(0, \pi^{2}\right)$, it is simple, $E(\lambda)$ is analytic at $\lambda=0$, and

$$
E(\lambda)=\pi^{2}-\pi^{4} \lambda^{2}\left(\int_{\mathbb{R}} f(x) d x\right)^{2}+O\left(\lambda^{3}\right) .
$$

This is the main result of this paper, which we'll prove in Section 2 using a calculation in the appendix. The technique used in our proof is closely patterned after the theory of bound states of $-\frac{d^{2}}{d x^{2}}+\lambda V(x)$ for $\lambda$ small as developed in [2], [10], [11], [14]. The key idea there is that $\left(-\frac{d^{2}}{d x^{2}}+k^{2}\right)^{-1}$ has a well-behaved limit as $k \downarrow 0$ except for a divergent rank one piece. In exactly the same way, $\left(-\Delta_{\Omega_{0}}^{D}-\pi^{2}+k^{2}\right)^{-1}$ has a nice limit as $k \downarrow 0$ except for a rank one piece.

Theorem 1.1 (or 1.2) leads to the following remarkable result which, roughly speaking, asserts that if on an arbitrarily small segment in the boundary $\partial \Omega_{0}$ of $\Omega_{0}$ the original Dirichlet boundary condition is replaced by a Neumann boundary condition, at least one additional eigenvalue is instantly created in the interval $\left(0, \pi^{2}\right)$.

Corollary 1.3. Let $\Omega_{0}=\mathbb{R} \times(0,1)$ and denote by $H(a, b)$ in $L^{2}\left(\Omega_{0}\right)$ the Laplacian on $\Omega_{0}$ with a Neumann boundary condition on the segment $(a, b) \times\{1\},-\infty<a<$ $b<\infty$, and Dirichlet boundary conditions on $\partial \Omega_{0} \backslash\{(a, b) \times\{1\}\}$. Then $H(a, b)$ has a discrete eigenvalue in $\left[\frac{\pi^{2}}{4}, \pi^{2}\right)$ no matter how small $|b-a|>0$ is.

Proof. Clearly $H(a, b) \geq 0$ and $\sigma_{\mathrm{ess}}(H(a, b))=\left[\pi^{2}, \infty\right)$. Enlarge $\Omega_{0}$ to $\Omega_{\lambda}$ of the type (2) with $\lambda>0$ sufficiently small and some $0 \leq f \in C^{\infty}(\mathbb{R})$ with $\operatorname{supp}(f)=$ $[a, b], f>0$ on $(a, b)$. By Theorem 1.1, $-\Delta_{\Omega_{\lambda}}^{D}$ has at least one eigenvalue $E_{\lambda} \in$ $\left(0, \pi^{2}\right)$. Next, decouple $\Omega_{0}$ and $\Omega_{\lambda} \backslash \Omega_{0}$ by a Neumann boundary condition along the segment $(a, b) \times\{1\}$. Denoting the resulting Laplace operator by $\hat{H}_{\Omega_{\lambda}}$, we obtain the direct sum decomposition $\hat{H}_{\Omega_{\lambda}}=H(a, b) \oplus-\widetilde{\Delta}(a, b)$ with respect to $L^{2}\left(\Omega_{\lambda}\right)=L^{2}\left(\Omega_{0}\right) \oplus L^{2}\left(\Omega_{\lambda} \backslash \Omega_{0}\right)$, where $-\widetilde{\Delta}(a, b)$ has Dirichlet (resp. Neumann) 
boundary conditions on $\partial \Omega_{\lambda} \backslash \partial \Omega_{0}$ (resp. $\left.(a, b) \times\{1\}\right)$. By Neumann decoupling (see, e.g., [12], p.270)

$$
0 \leq \inf \operatorname{spec}\left(\hat{H}_{\Omega_{\lambda}}\right) \leq \inf \operatorname{spec}\left(-\Delta_{\Omega_{\lambda}}^{D}\right) \leq E_{\lambda}<\pi^{2} .
$$

Choosing $f$ appropriately such that $\inf \operatorname{spec}(-\widetilde{\Delta}(a, b))>\pi^{2}$ (e.g., choose $f$ such that $\Omega_{\lambda} \backslash \Omega_{0}$ is a smoothed out rectangle of the type $(a, b) \times(1,1+c)$ with $0<c \ll$ $|b-a|)$, one obtains

$$
0 \leq \inf \operatorname{spec}(H(a, b)) \leq \inf \operatorname{spec}\left(-\Delta_{\Omega_{\lambda}}^{D}\right) \leq E_{\lambda}<\pi^{2} .
$$

That actually inf $\operatorname{spec}(H(a, b)) \geq \frac{\pi^{2}}{4}$ follows as in the proof of Corollary 1.4.

We note that the analogous result containing two such segments $(a, b)$ with Neumann boundary conditions placed symmetrically with respect to the axis $\mathbb{R} \times$ $\{1 / 2\}$ can be inferred from Lemma 3.2 in [5]. That paper also contains a variety of spectral results on acoustical waveguides (i.e., Neumann Laplacians as opposed to our Dirichlet Laplacians) using trial function techniques.

We have a number of remarks concerning Theorem 1.2:

(1) $\lambda \int_{\mathbb{R}} f(x) d x$ is exactly the area of $\Omega_{\lambda} \backslash \Omega_{0}$.

(2) In thinking about the higher-dimensional analogs, one needs to realize there are two independent dimensions in the above examples: the dimension of the cross section and the number of unbounded dimensions. In general, one can consider $K \subset \mathbb{R}^{n}$, a bounded connected open set, and $\Omega_{0}=\mathbb{R}^{\ell} \times K$. With minor changes, our analysis extends to general $(n, K)$ so long as $\ell=1$, that is, for $\Omega_{0}$ a long tube.

(3) In the notation of point (2), the results are $\ell$ dependent. For $\ell=2$, that is, $\Omega_{0}$ a long slab, there are still weakly coupled states, but as in [14], the binding is only $O\left(e^{-c / \lambda}\right)$. For $\ell \geq 3$, there will be no bound state if too small a bump is added.

(4) If one uses the one-dimensional Schrödinger operator [14] as a guide, one might guess that if $\int_{\mathbb{R}} f(x) d x=0$, then $-\Delta_{\Omega_{\lambda}}^{D}$ has a bound state for all sufficiently small $\lambda$; but since $-\Delta_{\Omega_{\lambda}}^{D}$ has second-order terms not found in the one-dimensional case, that is not totally clear.

(5) However, if $\int_{\mathbb{R}} f(x) d x<0$, then by our analysis, $-\Delta_{\Omega_{\lambda}}^{D}$ has no spectrum in $\left[0, \pi^{2}\right)$ if $\lambda$ is too small.

(6) Since $\Omega_{\lambda}$ isn't monotone if $f$ isn't positive, we cannot be sure that if $f$ is somewhere negative then $\Omega_{\lambda=1}$ has bound states even if $\int_{\mathbb{R}} f(x) d x>0$. Indeed, if $f$ is very close to -1 on a long region, we expect that $-\Delta_{\Omega_{\lambda=1}}^{D}$ has no bound states.

(7) We owe to Mark Ashbaugh the following observation:

Corollary 1.4. Let $\tilde{\Omega}=\{\mathbb{R} \times(0,2)\} \backslash\{\mathbb{R} \times\{1\}\} \cup\{(a, b) \times\{1\}\},-\infty<a<b<\infty$ (i.e., $\overline{\tilde{\Omega}}$ consists of two copies of $\Omega_{0}$ with the boundary between them removed in $(a, b) \times\{1\})$ and denote by $-\Delta_{\tilde{\Omega}}^{D}$ the associated Dirichlet Laplacian in $L^{2}(\tilde{\Omega})$. Then $-\Delta_{\tilde{\Omega}}^{D}$ has a discrete eigenvalue in $\left[\frac{\pi^{2}}{4}, \pi^{2}\right)$ independently of the size $|b-a|>0$ of the slit $(a, b) \times\{1\}$.

Proof. $\tilde{\Omega}$ has reflection symmetry under $y \rightarrow 2-y$. Thus, $-\Delta_{\tilde{\Omega}}^{D}$ is a direct sum of operators even and odd under this symmetry, and so $-\Delta_{\tilde{\Omega}}^{D} \cong H(a, b) \oplus-\Delta_{\Omega_{0}}^{D}$, where $H(a, b)$ is the operator in Corollary 1.3 (since even is equivalent to Neumann and odd to Dirichlet boundary conditions) and $\cong$ abbreviates unitary equivalence. 
Since $\sigma_{\text {ess }}(H(a, b))=\sigma_{\text {ess }}\left(-\Delta_{\Omega_{0}}^{D}\right)=\left[\pi^{2}, \infty\right)=\sigma_{\text {ess }}\left(-\Delta_{\tilde{\Omega}}^{D}\right)$, it suffices to prove that $-\Delta_{\tilde{\Omega}}^{D}$ has spectrum in $\left[\frac{\pi^{2}}{4}, \pi^{2}\right)$.

Let $\widehat{\Omega}=\Omega_{0} \cup\left\{(x, y) \in \mathbb{R}^{2} \mid a<x<b, 0<y<2\right\}$. Then $\widehat{\Omega} \subset \tilde{\Omega}$, so $\inf \operatorname{spec}\left(-\Delta_{\tilde{\Omega}}^{D}\right) \leq \inf \operatorname{spec}\left(-\Delta_{\tilde{\Omega}}^{D}\right)<\pi^{2}$ by Theorem 1.1. The lower bound then follows from $\tilde{\Omega} \subset \mathbb{R} \times(0,2)$.

Remark 1.5. An alternative proof of Theorem 1.1 can be based on the following trial function argument. Without loss of generality assume that $\Omega$ contains a small neighbourhood of the point $(0,1)$. Thus there are $a, b>0$ such that the triangle spanned by the points $(-a, 1),(a, 1)$ and $(0,1+b)$ is in $\Omega$. Define on $\Omega$

$$
\hat{\psi}_{\beta, \delta}(x, y)= \begin{cases}\sin (\pi y) e^{-\delta(|x|-a)}, & |x|>a, \quad 0<y<1, \\ \sin \left(\frac{\pi y}{1+\beta\left(1-\frac{|x|}{a}\right)}\right), & |x| \leq a, \quad 0<y<1+\beta\left(1-\frac{|x|}{a}\right), \\ 0, & \text { otherwise }\end{cases}
$$

where $0<\beta<b$ and $\delta>0$. This trial function certainly vanishes on $\partial \Omega$ and at $\infty$, and it is in the form domain $Q\left(-\Delta_{\Omega}^{D}\right)$. By a straightforward calculation we obtain

$$
E\left(\hat{\psi}_{\beta, \delta}\right)=\frac{\left(\nabla \hat{\psi}_{\beta, \delta}, \nabla \hat{\psi}_{\beta, \delta}\right)}{\left(\hat{\psi}_{\beta, \delta}, \hat{\psi}_{\beta, \delta}\right)}=\pi^{2}(1-2 a \delta \beta)+O\left(\beta^{2} \delta\right)+O\left(\delta^{2}\right)
$$

If we first choose $\beta$ and then $\delta$ small enough, we get

$$
E\left(\hat{\psi}_{\beta, \delta}\right)<\pi^{2}=\inf \sigma_{\text {ess }}\left(-\Delta_{\Omega}^{D}\right) .
$$

Since inf $\operatorname{spec}\left(-\Delta_{\Omega}^{D}\right)<E\left(\hat{\psi}_{\beta, \delta}\right)$ and $-\Delta_{\Omega}^{D}>0$, this proves Theorem 1.1. Note that $\sin (\pi y)$ in (4) represents the function $u(x, y)$ in (7) used prominently in Lemma 2.2 and in the proof of Theorem 1.2.

Spectral properties of quantum waveguides have received considerable attention recently. While a complete bibliography is beyond the scope of this paper, the interested reader is referred to [1], [3], [4], [5], [6], [7], [8], [13] and the literature cited therein. In particular, a weak coupling mechanism different from the one discussed in the present paper, based on arbitrarily small bending of tubes, has been studied in detail in [4] and [13].

Without entering into further details, we remark that Theorem 1.1 admits a variety of extensions. For instance, $\Omega$ and $\Omega_{0}$ need not coincide outside a sphere of radius $R$ as assumed in our condition (i); $\Omega$ only needs to approach $\Omega_{0}$ asymptotically (still assuming condition (ii)) since equality of the essential spectra of $-\Delta_{\Omega}^{D}$ and $-\Delta_{\Omega_{0}}^{D}$ as recorded in (1) is the crucial property in question. In addition, $\Omega$ could have various further branches running off to infinity as long as the asymptotic width of these branches is less than or equal to one in order to guarantee the validity of (1). Moreover, combining our results with the ones in [4] and [13] produces the same ground state effect for a bent tube of constant width one (and again additional bent branches running off to infinity of asymptotic widths not larger than one can be accommodated).

\section{§2. WEAK COUPLING ANALYSIS}

We'll study $-\Delta_{\Omega_{\lambda}}^{D}$ by a perturbation method. Since $L^{2}\left(\Omega_{\lambda}\right)$ is $\lambda$ dependent, it is difficult to use perturbation theory directly, so we'll map all the operators onto 
the same space. Let $U_{\lambda}: L^{2}\left(\Omega_{\lambda}\right) \rightarrow L^{2}\left(\Omega_{0}\right)$ be given by

$$
\left(U_{\lambda} \psi\right)(x, y)=\sqrt{1+\lambda f(x)} \psi(x,(1+\lambda f(x)) y) .
$$

Then $U_{\lambda}$ is unitary and

$$
H_{\lambda}=U_{\lambda}\left(-\Delta_{\lambda}^{D}\right) U_{\lambda}^{-1}-\pi^{2}
$$

acts in $L^{2}\left(\Omega_{0}\right)$. We subtract $\pi^{2}$ so that $\sigma_{\text {ess }}\left(H_{\lambda}\right)=[0, \infty)$.

A straightforward calculation found in the appendix (cf. (A.6)) proves that

$$
H_{\lambda}=H_{0}+\lambda \sum_{i=1}^{3} A_{i}^{*} B_{i}+\lambda^{2} \sum_{i=4}^{8} A_{i}^{*} B_{i},
$$

where each $A_{i}$ and $B_{i}$ is a first-order differential operator with coefficients which have compact support and ( $g$ is a $C^{\infty}$ function chosen such that $g \equiv 1$ on supp $f$ )

$$
\begin{aligned}
\text { (i) } & A_{1}^{*}=2 f(x) \frac{\partial}{\partial y}, & B_{1}=g(x) \frac{\partial}{\partial y}, \\
\text { (ii) } & A_{2}^{*}=f^{\prime \prime}(x), & B_{2}=g(x)\left(y \frac{\partial}{\partial y}+\frac{1}{2}\right), \\
\text { (iii) } & A_{3}^{*}=\left(2 y \frac{\partial}{\partial y}+1\right) g(x), & B_{3}=f^{\prime}(x) \frac{\partial}{\partial x}
\end{aligned}
$$

(we'll see below that to leading order only $A_{1}^{*} B_{1}$ matters).

Rewrite $(5)$ as follows. Define $C(\lambda), D: L^{2}\left(\Omega_{0}\right) \rightarrow L^{2}\left(\Omega_{0}\right) \otimes \mathbb{C}^{8}$ by

$$
\begin{array}{ll}
(C \varphi)_{i}= \begin{cases}A_{i} \varphi, & i=1,2,3, \\
\lambda A_{i} \varphi, & i=4, \ldots, 8,\end{cases} \\
(D \varphi)_{i}=B_{i} \varphi, & i=1, \ldots, 8 .
\end{array}
$$

Then (5) becomes

$$
H_{\lambda}=H_{0}+\lambda C^{*}(\bar{\lambda}) D
$$

At this point we can apply Birman-Schwinger-type techniques (see, e.g., [12], Sect. XIII.3).

Lemma 2.1. Let $k \in \mathbb{C}, \operatorname{Re} k>0$ and $\lambda \in \mathbb{R}$. Then $-k^{2}$ is an eigenvalue of $H_{\lambda}$ if and only if

$$
\lambda D\left(H_{0}+k^{2}\right)^{-1} C^{*} \equiv Q_{\lambda}
$$

has -1 as an eigenvalue.

Proof. If $Q_{\lambda} \psi \equiv-\psi$, then $-\lambda\left(H_{0}+k^{2}\right)^{-1} C^{*} \psi \equiv \varphi$ is seen to satisfy $H_{\lambda} \varphi=-k^{2} \varphi$. Conversely, if $H_{\lambda} \varphi=-k^{2} \varphi$, then $\varphi \in Q\left(H_{\lambda}\right) \subset D(D)$, so $\psi=D \varphi$ is in $L^{2}\left(\Omega_{0}\right)$ and $Q_{\lambda} \psi=-\psi$.

Lemma 2.2. Let $h$ be a $C^{\infty}$ function of compact support in $\mathbb{R}$. Then

$$
h\left(H_{0}+k^{2}\right)^{-1} h=\frac{(h u, \cdot) h u}{2 k}+A(k),
$$

where $u$ is the function

$$
u(x, y)=2^{\frac{1}{2}} \sin (\pi y)
$$


and $A(k)$ is a bounded operator-valued function of $k$, which can be analytically continued from $\{k \in \mathbb{C} \mid \operatorname{Re} k>0\}$ to a region that includes $k=0$. Indeed, even $\left(H_{0}+1\right)^{1 / 2} A(k)\left(H_{0}+1\right)^{1 / 2}$ has an analytic continuation into such a region.

Moreover, $\left(H_{0}+1\right)^{1 / 2} A(k)\left(H_{0}+1\right)^{1 / 2}$ is bounded uniformly in $\{k \in \mathbb{C}|| \operatorname{Arg} k \mid<$ $\pi / 3\} \cup\{$ a small disk about $k=0\}$ ( $\frac{\pi}{3}$ can be replaced by any number strictly less than $\left.\frac{\pi}{2}\right)$.

Proof. Let $\mathcal{H}_{0} \subset L^{2}\left(\Omega_{0}\right)$ be the space of $L^{2}\left(\Omega_{0}\right)$ functions of the form $\varphi(x) \sin (\pi y)$, $\sin (\pi y)$ being chosen as the lowest eigenfunction of $\left(-\frac{d^{2}}{d y^{2}}\right)^{D}$. Let $P_{0}$ be the projection onto $\mathcal{H}_{0}$. Then $\left(H_{0}+k^{2}\right)^{-1}\left(1-P_{0}\right)$ has an analytic continuation into the region $\left\{k \in \mathbb{C} \mid-k^{2} \in \mathbb{C} \backslash\left[3 \pi^{2}, \infty\right)\right\}$, since the lowest point in the spectrum of $H_{0}\left(1-P_{0}\right) \uparrow\left(1-P_{0}\right) L^{2}\left(\Omega_{0}\right)$ is $3 \pi^{2}$.

On the other hand, $h\left(H_{0}+k^{2}\right)^{-1} P_{0} h$ has the explicit integral kernel

$$
(2 k)^{-1} h(x) h\left(x^{\prime}\right) u(y) u\left(y^{\prime}\right) e^{-k\left|x-x^{\prime}\right|}=a_{1}(k)+a_{2}(k),
$$

where $a_{1}(k)$ is obtained by replacing $e^{-k\left|x-x^{\prime}\right|}$ by 1 and $a_{2}(k)$ by using $e^{-k\left|x-x^{\prime}\right|}-1$ in its place. The first term is the explicit rank one piece in (6) and the second term is analytic as a Hilbert-Schmidt kernel at $k=0$.

It is easy to modify this argument to accommodate the extra factors of $\left(H_{0}+1\right)^{1 / 2}$ and prove the boundedness.

Proof of Theorem 1.2. Consider first the operator on $L^{2}\left(\Omega_{0}\right) \otimes \mathbb{C}^{8}$ :

$$
L_{0}=(C(\lambda=0) u, \cdot) D u,
$$

where $u$ is given by $(7)$. Then $L_{0}$ is a rank one operator, so it has a single eigenvalue at

$$
e_{0}=\operatorname{Tr}\left(L_{0}\right)
$$

But $C_{i}(0)=0$ for $i=4, \ldots, 8, B_{3} u=0$, and $\left(A_{2} u, B_{2} u\right)=0$ since $\int_{\mathbb{R}} f^{\prime \prime}(x) d x=0$. It follows that

$$
\begin{aligned}
e_{0}=\left(A_{1} u, B_{1} u\right) & =-2\left(\int_{\mathbb{R}} f(x) d x\right) \int_{0}^{1} 2 \pi^{2} \cos ^{2}(\pi y) d y \\
& =-2 \pi^{2} \int_{\mathbb{R}} f(x) d x .
\end{aligned}
$$

Let $k=\lambda \ell$. Then by Lemma 2.2 ,

$$
\lambda D\left(H_{0}+k^{2}\right)^{-1} C^{*}=Q(\lambda, \ell)
$$

has the form

$$
Q(\lambda, \ell)=\frac{1}{2 \ell} L_{\lambda}+\lambda M(\lambda, \ell)
$$

where

(i) $L_{\lambda}$ is rank one and $L_{\lambda}=L_{0}+\lambda \tilde{L}$.

(ii) $M(\lambda, \ell)=D A(\lambda, \ell) C^{*}$, where $A$ is given by Lemma 2.2 and $h$ is chosen such that $h \equiv 1$ in a neighborhood of $\operatorname{supp} f$.

By Lemma 2.2, we are interested in when $Q(\lambda, \ell)$ has -1 as an eigenvalue for $\lambda>0$ and $\ell>0$. Since $M$ is uniformly bounded in $\lambda$ on a sector about $(0, \infty)$, this can happen where $\lambda$ is small if $\frac{e_{0}}{2 \ell}$ is near -1 , that is, $\ell$ is near $\frac{-e_{0}}{2}>0$ since $\int_{\mathbb{R}} f(x) d x>0$ by hypothesis. 
For such $\ell$ and $\lambda$ small, $Q(\lambda, \ell)$ has exactly one eigenvalue near -1 , call it $E(\lambda, \ell)$, which by eigenvalue perturbation theory ([9], Ch.2, [12], Ch.XII) is jointly analytic in $\lambda, \ell$. Let

$$
F(\lambda, \ell)=2 \ell(E(\lambda, \ell)+1)
$$

Since $\left.2 \ell Q(\lambda, \ell)\right|_{\lambda=0}$ is independent of $\ell$,

$$
\left.\frac{\partial(2 \ell E(\lambda, \ell))}{\partial \ell}\right|_{\lambda=0}=0 \quad \text { and so }\left.\frac{\partial F(\lambda, \ell)}{\partial \ell}\right|_{\lambda=0, \ell=-e_{0} / 2}=2 \neq 0
$$

It follows by the implicit function theorem that for $\lambda$ sufficiently small, there is an analytic function $\ell(\lambda)=-\frac{e_{0}}{2}+O(\lambda)$ so that for $\lambda>0$ and $\ell$ in the sector $|\operatorname{Arg} \ell|<\frac{\pi}{3},-1$ is an eigenvalue of $Q(\lambda, \ell)$ if and only if $\ell=\ell(\lambda)$. Since $H_{\lambda}$ for $\lambda$ real has only real eigenvalues, $\ell(\lambda)$ must be real for $\lambda>0$. Thus $H_{\lambda}$ has a unique eigenvalue, $e(\lambda)$, in $(-\infty, 0)$, given by $e(\lambda)=-\lambda^{2}\left(-\frac{e_{0}}{2}\right)^{2}+O\left(\lambda^{3}\right)$, as claimed.

\section{ACKNOWLEDGMENTS}

It is a pleasure to thank Mark Ashbaugh and Thomas Hoffmann-Ostenhof for numerous discussions on this subject. We also thank Brian Davies for making us aware of reference [5]. W. B. is indebted to the Department of Mathematics of the University of Missouri, Columbia for the hospitality extended to him during a stay in the spring of 1994. Furthermore, he gratefully acknowledges the financial support for this stay by the Technische Universität Graz, Austria.

$$
\text { Appendix: Calculating } \widetilde{H}_{\lambda}=U_{\lambda}\left(-\Delta_{\Omega_{\lambda}}^{D}\right) U_{\lambda}^{-1} \text {. }
$$

We use coordinates $(x, y)$ on $\Omega_{0}$ and $(s, u)$ on $\Omega_{\lambda}$. Thus $U_{\lambda}$ becomes

$$
\begin{aligned}
U_{\lambda}: & L^{2}\left(\Omega_{\lambda}\right) \\
\tilde{\psi}(s, u) & \mapsto L^{2}\left(\Omega_{0}\right) \\
& \mapsto \psi(x, y)=\sqrt{1+\lambda f(x)} \tilde{\psi}(x,(1+\lambda f(x)) y) .
\end{aligned}
$$

The coordinate transformation is

$$
x=s, \quad y=(1+\lambda f(s))^{-1} u .
$$

The form associated with $-\Delta_{\Omega_{\lambda}}^{D}$ is given by

$$
\begin{aligned}
q_{\Omega_{\lambda}}^{D}: Q\left(-\Delta_{\Omega_{\lambda}}^{D}\right) \times Q\left(-\Delta_{\Omega_{\lambda}}^{D}\right) & \rightarrow \mathbb{C} \\
(\varphi, \psi) & \mapsto(\nabla \varphi, \nabla \psi),
\end{aligned}
$$

where $Q\left(-\Delta_{\Omega_{\lambda}}^{D}\right)=H_{0}^{1,2}\left(\Omega_{\lambda}\right)$ is the usual Sobolev space (i.e., the completion of $C_{0}^{\infty}$ under the norm $\left.\|\cdot\|_{\nabla}=\left(\|\nabla \cdot\|^{2}+\|\cdot\|^{2}\right)^{1 / 2}\right)$. By unitary equivalence, the quadratic form associated with $\widetilde{H}_{\lambda}$ is

$$
\begin{aligned}
& q_{\widetilde{H}_{\lambda}}: \quad U_{\lambda} Q\left(-\Delta_{\Omega_{\lambda}}^{D}\right) \times U_{\lambda} Q\left(-\Delta_{\Omega_{\lambda}}^{D}\right) \rightarrow \mathbb{C} \\
& (\varphi, \psi) \quad \mapsto q_{\Omega_{\lambda}}^{D}\left(U_{\lambda}^{-1} \varphi, U_{\lambda}^{-1} \psi\right) .
\end{aligned}
$$

The form domain of $\widetilde{H}_{\lambda}$ is $U_{\lambda} H_{0}^{1,2}\left(\Omega_{\lambda}\right)=\overline{U_{\lambda} C_{0}^{\infty}\left(\Omega_{\lambda}\right)}$, where the bar denotes completion under the norm $\|\cdot\|_{q}=\left(q_{\widetilde{H}_{\lambda}}(\cdot, \cdot)+\|\cdot\|^{2}\right)^{1 / 2}$. 
Next we calculate the quadratic form $q_{\widetilde{H}_{\lambda}}(\varphi, \psi)$ for $\varphi, \psi \in C_{0}^{\infty}\left(\Omega_{0}\right)$. We use the shorthand $c(x)=1+\lambda f(x)$ and use subscripts to denote partial derivatives.

$$
\begin{aligned}
& q_{\widetilde{H}_{\lambda}}(\varphi, \psi)=q_{\Omega_{\lambda}}^{D}\left(U_{\lambda}^{-1} \varphi, U_{\lambda}^{-1} \psi\right) \\
& =\int_{\Omega_{\lambda}}\left(\partial_{s} c(s)^{-1 / 2} \overline{\varphi\left(s, \frac{u}{c(s)}\right)}\right)\left(\partial_{s} c(s)^{-1 / 2} \psi\left(s, \frac{u}{c(s)}\right)\right) d s d u \\
& +\int_{\Omega_{\lambda}}\left(\partial_{u} c(s)^{-1 / 2} \overline{\varphi\left(s, \frac{u}{c(s)}\right)}\right)\left(\partial_{u} c(s)^{-1 / 2} \psi\left(s, \frac{u}{c(s)}\right)\right) d s d u \\
& =\int_{\Omega_{0}}\left\{\left[-\frac{1}{2} \frac{c^{\prime}(x)}{c(x)} \overline{\varphi(x, y)}+\overline{\varphi_{x}(x, y)}-\frac{y c^{\prime}(x)}{c(x)} \overline{\varphi_{y}(x, y)}\right]\right. \\
& {\left[-\frac{1}{2} \frac{c^{\prime}(x)}{c(x)} \psi(x, y)+\psi_{x}(x, y)-\frac{y c^{\prime}(x)}{c(x)} \psi_{y}(x, y)\right]} \\
& \left.+\frac{1}{c(x)^{2}} \overline{\varphi_{y}(x, y)} \psi_{y}(x, y)\right\} d x d y \\
& =\int_{\Omega_{0}}\left\{\overline{\varphi_{x}(x, y)} \psi_{x}(x, y)+\frac{1+y^{2} c^{\prime}(x)^{2}}{c(x)^{2}} \overline{\varphi_{y}(x, y)} \psi_{y}(x, y)\right. \\
& -\frac{y c^{\prime}(x)}{c(x)}\left(\overline{\varphi_{x}(x, y)} \psi_{y}(x, y)+\overline{\varphi_{y}(x, y)} \psi_{x}(x, y)\right) \\
& -\frac{c^{\prime}(x)}{2 c(x)}\left(\overline{\varphi_{x}(x, y)} \psi(x, y)+\overline{\varphi(x, y)} \psi_{x}(x, y)\right) \\
& +\frac{y c^{\prime}(x)^{2}}{2 c(x)^{2}}\left(\overline{\varphi_{y}(x, y)} \psi(x, y)+\overline{\varphi(x, y)} \psi_{y}(x, y)\right) \\
& \left.+\frac{c^{\prime}(x)^{2}}{4 c(x)^{2}} \overline{\varphi(x, y)} \psi(x, y)\right\} d x d y \text {. }
\end{aligned}
$$

By partial integration we get the operator

$$
\begin{aligned}
\widetilde{H}_{\lambda}= & -\frac{\partial^{2}}{\partial x^{2}}-\frac{1+y^{2} \lambda^{2} f^{\prime}(x)^{2}}{c(x)^{2}} \frac{\partial^{2}}{\partial y^{2}}+\left(\frac{y \lambda f^{\prime \prime}(x)}{c(x)}-\frac{3 y \lambda^{2} f^{\prime}(x)^{2}}{c(x)^{2}}\right) \frac{\partial}{\partial y} \\
& +\frac{2 y \lambda f^{\prime}(x)}{c(x)} \frac{\partial}{\partial x} \frac{\partial}{\partial y}+\frac{\lambda f^{\prime}(x)}{c(x)} \frac{\partial}{\partial x}+\frac{\lambda f^{\prime \prime}(x)}{2 c(x)}-\frac{3 \lambda^{2} f^{\prime}(x)^{2}}{4 c(x)^{2}} \\
= & -\Delta_{\Omega_{0}}^{D}+\lambda\left[2 f(x) \frac{\partial^{2}}{\partial y^{2}}+y f^{\prime \prime}(x) \frac{\partial}{\partial y}+2 y f^{\prime}(x) \frac{\partial}{\partial x} \frac{\partial}{\partial y}+f^{\prime}(x) \frac{\partial}{\partial x}+\frac{f^{\prime \prime}(x)}{2}\right] \\
- & \lambda^{2}\left[\frac{3 f(x)^{2}+2 \lambda f(x)^{3}+y^{2} f^{\prime}(x)^{2}}{(1+\lambda f(x))^{2}} \frac{\partial^{2}}{\partial y^{2}}+\left(\frac{y f(x) f^{\prime \prime}(x)}{(1+\lambda f(x))}+\frac{3 y f^{\prime}(x)^{2}}{(1+\lambda f(x))^{2}}\right) \frac{\partial}{\partial y}\right. \\
+ & \left.\frac{2 y f(x) f^{\prime}(x)}{(1+\lambda f(x))} \frac{\partial}{\partial x} \frac{\partial}{\partial y}+\frac{f(x) f^{\prime}(x)}{(1+\lambda f(x))} \frac{\partial}{\partial x}+\frac{f(x) f^{\prime \prime}(x)}{2(1+\lambda f(x))}+\frac{3 f^{\prime}(x)^{2}}{4(1+\lambda f(x))^{2}}\right] .
\end{aligned}
$$

Since we assumed $f \in C_{0}^{\infty}(\mathbb{R})$, clearly $C_{0}^{\infty}\left(\Omega_{0}\right) \subset D\left(\widetilde{H}_{\lambda}\right)=U_{\lambda} D\left(-\Delta_{\Omega_{\lambda}}^{D}\right)$. 
Actually, $U_{\lambda} C_{0}^{\infty}\left(\Omega_{\lambda}\right)=C_{0}^{\infty}\left(\Omega_{0}\right)$. From (A.5) we infer that the norm $\|\cdot\|_{q}$ is equivalent to the norm $\|\cdot\|_{\nabla}$, i.e.,

$$
Q\left(\widetilde{H}_{\lambda}\right)=\overline{U_{\lambda} C_{0}^{\infty}\left(\Omega_{\lambda}\right)}=H_{0}^{1,2}\left(\Omega_{0}\right) .
$$

\section{REFERENCES}

[1] M.S. Ashbaugh and P. Exner, Lower bounds to bound state energies in bent tubes, Phys. Lett. A 150 (1990), 183-186. MR 91h:35235

[2] R. Blankenbecler, M.L. Goldberger, and B. Simon, The bound states of weakly coupled longrange one-dimensional quantum Hamiltonians, Ann. Phys. 108 (1977), 69-78. MR 56:14250

[3] P. Duclos and P. Exner, Curvature vs. thickness in quantum waveguides, Czech. J. Phys. 41 (1991), 1009-1018; erratum, vol. 42, 1992, p. 344.

[4] P. Duclos and P. Exner, Curvature-induced bound states in quantum waveguides in two and three dimensions, Rev. Math. Phys. 7 (1995), 73-102. MR 95m:81239

[5] D. V. Evans, M. Levitin, and D. Vassiliev, Existence theorems for trapped modes, J. Fluid Mech. 261 (1994), 21-31. MR 94m:76113

[6] P. Exner, Bound states in quantum waveguides of a slowly decaying curvature, J. Math. Phys. 34 (1993), 23-28. MR 94d:81038

[7] P. Exner and P. Šeba, Bound states in curved quantum waveguides, J. Math. Phys. 30 (1989), 2574-2580. MR 90i:81194

[8] P. Exner, P.Šeba, and P. Šťovíček, On existence of a bound state in an L-shaped waveguide, Czech. J. Phys. B 39 (1989), 1181-1191.

[9] T. Kato, Perturbation Theory for Linear Operators, corr. printing of the 2nd ed., Springer Verlag, Berlin, 1976. MR 53:11389

[10] M. Klaus, On the bound state of Schrödinger operators in one dimension, Ann. Phys. 108 (1977), 288-300. MR 58:20010

[11] M. Klaus and B. Simon, Coupling constant thresholds in nonrelativistic quantum mechanics. I: Short-range two-body case, Ann. Phys. 130 (1980), 251-281. MR 82h:81028a

[12] M. Reed and B. Simon, Methods of Modern Mathematical Physics. IV. Analysis of Operators, Academic Press, New York, 1978. MR 58:12429c

[13] W. Renger and W. Bulla, Existence of bound states in quantum waveguides under weak conditions, Lett. Math. Phys. 35 (1995), 1-12. MR 96g:81052

[14] B. Simon, The bound state of weakly coupled Schrödinger operators in one and two dimensions, Ann. Phys. 97 (1976), 279-288. MR 53:8646

(W. Bulla) Institute for Theoretical Physics, Technical University of Graz, A-8010 Graz, Austria

E-mail address: bulla@itp.tu-graz.ac.at

(F. Gesztesy and W. Renger) Department of Mathematics, University of Missouri, Columbia, Missouri 65211

E-mail address, F. Gesztesy: fritz@math.missouri.edu

E-mail address, W. Renger: walter@mumathnx3.cs.missouri.edu

(B. Simon) Division of Physics, Mathematics, and Astronomy, California Institute of Technology, Pasadena, California 91125 